\title{
MEASUREMENT OF INSECT FLIGHT FORCES USING A MEMS BASED PHYSICAL SENSOR
}

\author{
Mansoor Nasir a, Michael Dickinson ${ }^{b}$ and Dorian Liepmann ${ }^{a}$ \\ ${ }^{a}$ Dept. of Bioengineering, Berkeley Sensors and Actuator Center, University of California at Berkeley, USA \\ ${ }^{\mathrm{b}}$ Bioengineering, California Institute of Technology, Pasadena, CA ,USA
}

\begin{abstract}
In this paper we discuss the development of a force sensor for investigation of flight forces in flying insects. The proposed sensor uses piezoresistive sensing techniques to quantitatively measure changes in flight forces of a tethered insect. The sensor is fabricated on a wafer-level using standard MEMS technology. The goal of the research is the development of a sensor that allows for the measurement of forces with more than one degree of freedom (DOF). Direct measurement of rotational and translational forces will be very useful in elucidating complex aerodynamics mechanisms due to rapidly rotating and flapping wings, the understanding of which is critical in the development of microflying robotic insects.
\end{abstract}

\section{INTRODUCTION}

Flying insects are able to perform remarkable maneuvers and flying acrobats by a deceptively simple process of beating their wings. However, a closer look at insect flight reveals a highly complex and sophisticated mechanism based on unsteady aerodynamics. These mechanisms include principles like rotational circulation, delayed stall and wake capture which allow the insects to generate flight forces responsible for keeping them aloft. Not only do insects have more independent force controls than fixed wing aircrafts but the flight forces vary both in frequency and amplitude. Adding to the complexity is the fact that there is a huge diversity of flying insects with different wing morphologies. The study of insect flight is therefore a challenging problem from both, engineering and biological perspectives.

Biologists have generally focused on a selected few biologically significant flying insects to study insect flight. Of these fruit flies (Drosphila melanogaster) is probably one of the most frequently used model [1]. House flies (Musca domestica) and hawkmoth (Manduca sexta) have also been used for scientific research [2], [3]. These represent superb models for elucidating general principles that are critical in the realization of any biomemtics inspired flying microrobotic systems.

By far the most important tool for understanding flight mechanisms thus far has been high speed videography. By breaking down the rapidly beating wing motion into separate frames and then applying image processing tools, changes in wing orientation and wingbeat frequency and amplitude can be studied. Since the insects are small and hard to observe in free-flight, Dickenson et. al. developed a virtual reality flight arena where they study tethered simulation of insect flight. A wing beat analyzer uses optical tracking of tethered fruit flies to deduce changes in wing beat frequency and amplitude and this method has been successfully used to make yaw force measurements [4]. However, optical tracking methods require elaborate setups and it is very difficult to simultaneously measure rotational and translational forces in real time. Therefore, quantitative force measurements can greatly assist scientist in understanding insect flight both from neurophysiological and engineering standpoints.

MEMS sensors are being increasingly used in biological investigations because they combine the advantages of integrated circuit microfabrication techniques with robust macroscopic design and instrumentation principles, thus allowing an easier interface with biological environments ranging anywhere from single-cell manipulation to wafer-level drug delivery systems.
Consequently, MEMS force sensor are ideal for measuring insect flight forces which requires a design robust enough to support the insect weight but at the same time is sensitive enough to measure forces in $\mathrm{mN}-\mu \mathrm{N}$ range. Forces can be measured using pieozoresistive, capacitive, optical and piezoelectric sensing techniques, with each having its advantages and drawbacks. However because of the aforementioned requirements, piezoresistive sensing is most suitable for measuring multidirectional forces as it provides ease of design, fabrication and needs simpler backend electronics. It should be mentioned however that capacitive force sensing has been successfully shown to accurately measure lift forces in fruit flies [5]. Still multi DOF force measurement remains a challenging problem yet to be solved with any kind of sensing mechanism.

The proposed force sensor uses piezoresistive sensing techniques to measure instantaneous flight force. Concurrent research on a multi DOF is also being conducted and the initial results have been reported [6]. Recently other biological applications of MEMS strain gauge force sensors have been reported in literature as well [7].

\section{FORCE MICROSENSOR DESIGN}

Piezoresistivity is the change in resistance of the material when it is under stress. By tactically placing strain gauges at high stress areas of a structure, force can be measured. Semiconductors strain gauges are not only well-suited for bulk fabrication but are up to two orders more sensitive than metal counterparts. Since the underlining theory is well understood and the sensing mechanism is inherently mechanical, novel designs can be used measure multidirectional forces with increased sensitivity.

In developing a microsensor for measurement of flight forces, a number of requirements need to be satisfied. The sensor has to balance the material strength needed to support the weight of the flying insect while at the same time remain compliant enough to measure forces in the desired range. The design needs to be able to allow easy tethering of the fly and placement inside the virtual flight arena. Sensor reusability is important for reliable and repeatable experimental test results. Fig. 1 shows a solid model of the force sensor that replaces the current tether.

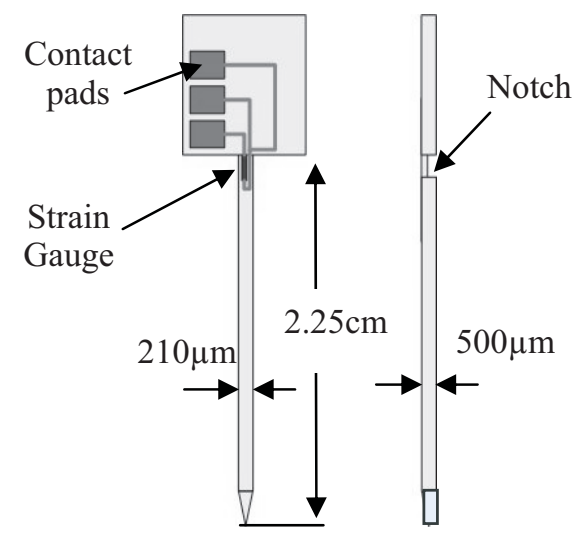

FIGURE 1: A model of force sensor. The fly is tethered at the tapered end of the sensor. 
This design has only one strain gauge and therefore it cannot resolve force into its components. The wider section is glued to a printed circuit board and the contact pads are used for electrical interface with the PCB. Linear beam theory implies that the maximum strain is near the base of the cantilever and so placing a strain gauge in this area provides higher force sensitivity. In addition to this the substrate under the strain gauge is thinner than the rest of beam. Since most of the beam bending is restricted to this section it acts like a stress-riser and further increases our force sensitivity. Sensor sensitivity is controllable via fabrication techniques allowing for a wide range of forces and frequencies to be measurements.

Strain gauges are most commonly used in Wheatstone bridge configurations. The bridge is initially balanced with the strain gauges in the circuitry without any force applied. Once acted upon by a force, gauge resistance changes and the unbalanced bridge results in an output voltage which can then be amplified and filtered.

\section{ANALYTICAL MODEL}

Linear beam theory can be used to find the governing equations defining the relation between the force acting at the tip of the sensor and resulting strain across each strain gauges. The base of the sensor is essentially an anchor and therefore the forces only deform the beam.

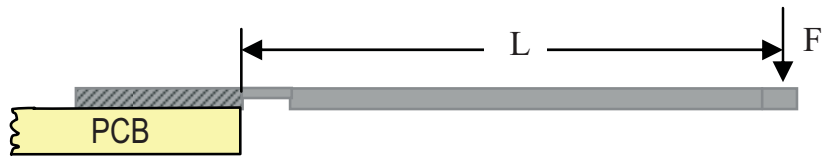

FIGURE 2: Side view of the sensor with ' $L$ ' being the length between the point where force $F$ is applied and the anchor.

If the strain gauge is located on the top surface of the thinner section of the beam then the stress is given by

$$
\sigma=\frac{M}{I} c \quad \text { where } \quad I=\frac{b h^{3}}{12}
$$

where $c$ is the distance from neutral fiber. $I$, which is the moment of inertia, depends on the thickness of the beam $h$, at the point where strain gauge is located. Using the Hook's law the change in resistance of the strain gauge can be related to the force being applied at the tip of the beam.

$$
\begin{gathered}
\sigma=\varepsilon . \mathrm{E} \\
\therefore \frac{\Delta R}{R}=G \frac{\text { L.F.c }}{b h^{3} / 12} \quad \varepsilon=\frac{1}{G} \frac{\Delta R}{R}
\end{gathered}
$$

where $E$, the Young's Modulus and $G$, the gauge factor are material properties for the strain gauge. We can see that by decreasing $h$ the stress in the thinner section can be greatly increased. Thus the change in resistance due to piezoresistive effect is magnified as seen in finite element simulation shown in Fig. 3. Also the stiffer the beam, the higher is the resonant frequency which is an important factor for testing insects with higher wingbeat frequencies.

This simple analytical model is only shown for the one force. Forces in other directions contribute to the overall change of resistance of the strain gauge but since there is only one strain gauge in this case, there is no way to resolve for these components in this design.

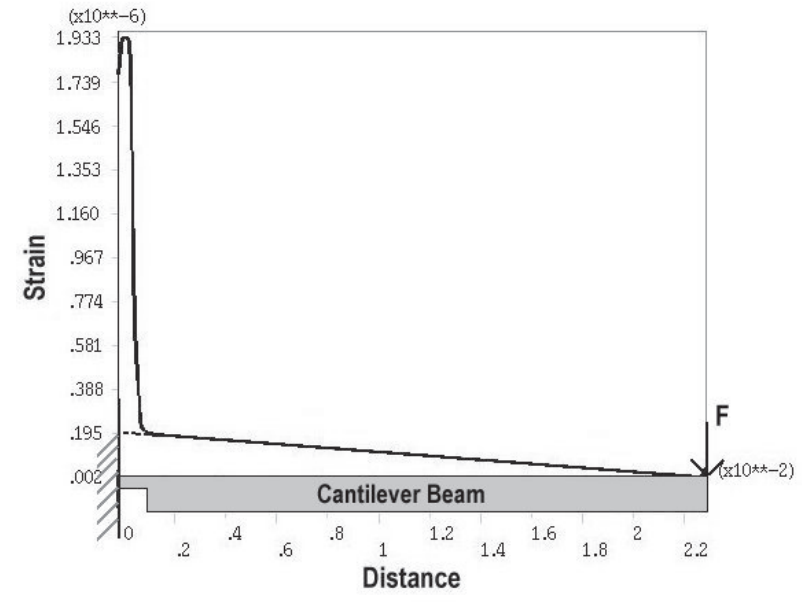

FIGURE 3: Simulation shows that by making a section of the beam near the anchor thinner, $160 \mu \mathrm{m}$ as opposed to $500 \mu \mathrm{m}$, strain was magnified nearly10-fold.

\section{FABRICATION}

Standard bulk micromachining was used to fabricate the force sensor. The fabrication process has been standardized and requires only four masks.

A single p-doped polysilicon strain gauge is microfabricated near the base of the cantilever beam with Al leads for measuring the voltages across it. This sensor has three wires coming off the strain gauge to allow the strain gauge to be hooked up in a threewire $1 / 4$ Wheatstone bridge which provides better temperature compensation. Lead-gauge overlap helps reduce the contact resistance. Low temperature oxide (LTO) deposited over the Al leads not only protects them from getting scratched but also acts as a mask for the final etch done from the top side (Fig. 4, 5).

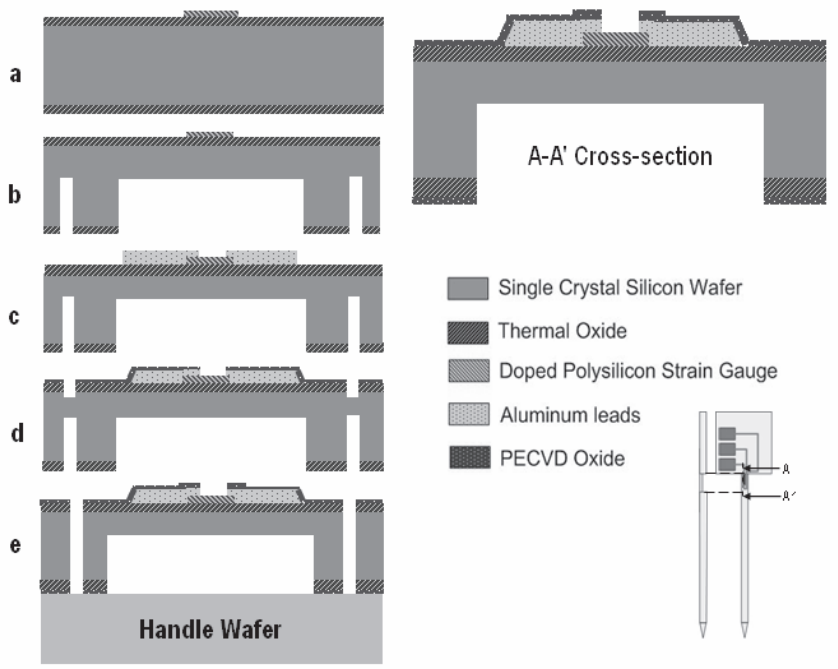

FIGURE 4: (a) A oxide passivation layer $(<1 \mu \mathrm{m})$ is grown on a P-type single crystal Si wafer followed by deposition, annealing and pattering of $N$-doped polysilicon layer ( 400A (b) DRIE etch is used to etch from the back side to thin the substrate under the strain gauge and also in the trenches for through-etch (c) Aluminum (Al) $(\sim 1.2 \mu \mathrm{m})$ is sputtered and then patterned using wet etch.(d) A thin layer of LTO $(0.5 \mu \mathrm{m})$ is deposited over the Al leads for protection. This oxide layer is etched in a RIE-etcher to open holes for through-etch (e) After putting on a handle wafer, a final DRIE-etch is done from the front side to release the sensors from the Si wafer. 


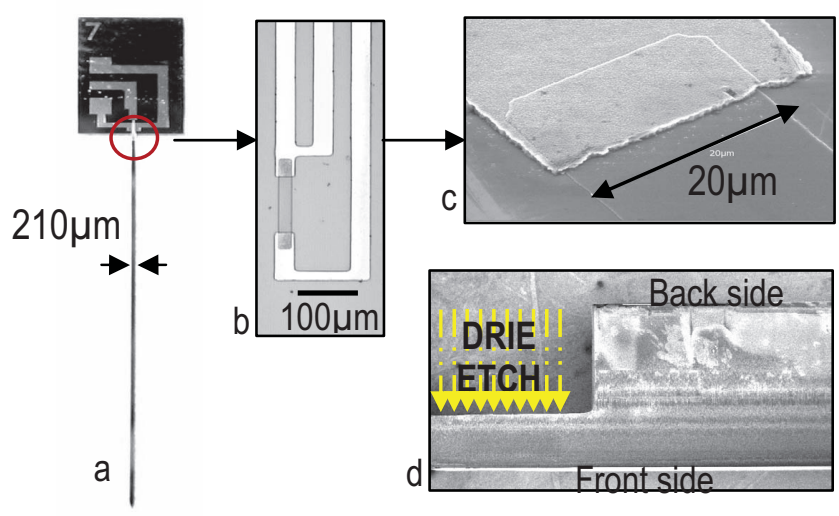

FIGURE 5: (a) Microfabricated force sensor with one strain gauge. (b) Magnified image shows the doped poly strain gauge and the Al leads. (c) SEM of the strain gauge and Al lead overlapping it (d) Side view SEM of the sensor shows the notch made by DRIE etching from the backside.

The electrical interface for the sensor is very simple and is made by gluing the sensor to the PCB and then using ultrasonic wirebonder to connect the pads to PCB connects.

\section{SENSOR CALIBRATION}

The force sensor was calibrated for both static and dynamic loads. The strain gauge is connected in a three-wire quarter Wheatstone bridge. The output is amplified, filtered ( $1 \mathrm{kHz}$ cutoff) and digitized to be recorded by software. For static loading, dead weights were used to apply force at the tip of the sensor. The bridge is first balanced sensor with no weight and then once loaded the change in voltage is recorded. By loading the sensor with different weights a relationship between force and voltage can be found (Fig. 6)

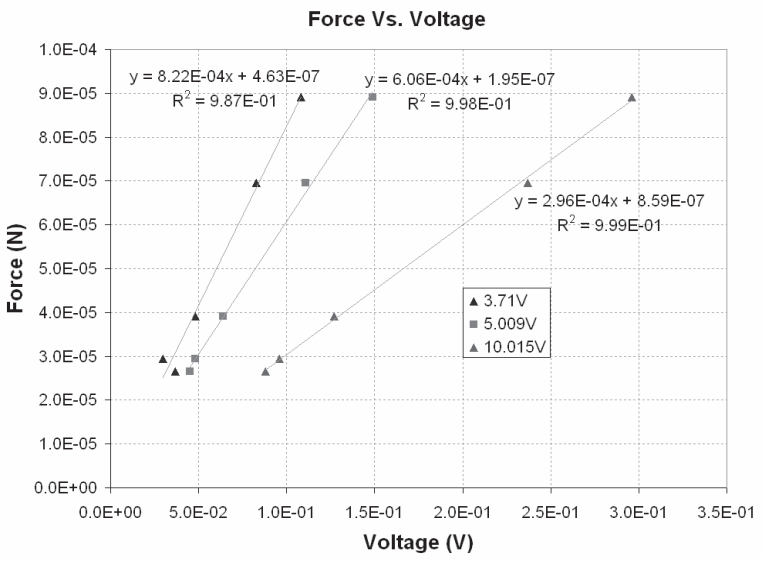

FIGURE 6: At different bridge input voltages the strain gauge voltage follows different curves due to self-heating effects in strain gauge which becomes more prominent with higher currents (i.e. bridge input voltage). However the relationship to force is still linear in each case.

A nanoindenter machine was used to find the spring constant of the sensor and it was within reasonable error of the values predicted by FEM simulations. Since flying insects are beating their wings during flight, the sensor must be tested under dynamic loading conditions as well (Fig. 7).

The resonant frequency was found by looking at the impulse response of the beam. It was verified that the resonant frequency of the beam is higher than the wingbeat frequencies of commonly studied insect, which are typically in $100-200 \mathrm{~Hz}$ range [8]. This way aliasing effects due to under sampling can be avoided.

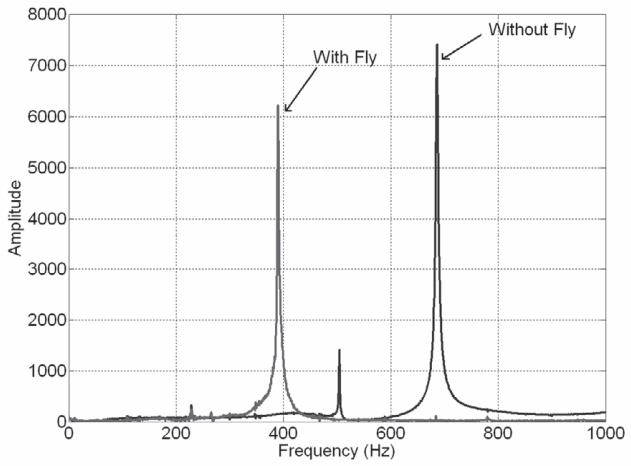

FIGURE 7: Even when the beam is weighed down with a tethered house fly, the resonant frequency is still higher than the wingbeat frequency of the fly, reported to be around $190 \mathrm{~Hz}$ [9].

\section{INSTANTANEOUS FLIGHT FORCE MEASUREMENT}

Once calibrated, flight forces in simulated flight can be found by tethering the insect to the end of the force sensor. The results shown are recorded for force measurements with tethered house flies. In order to tether the fly, it is temporarily sedated by refrigeration for 5 minutes. While sedated, the beam is glued to the fly in the thorax region. The flies are glued at a slight angle to the horizontal to mimic the body orientation in free-flight. As the body temperature of the fly returns to normal it 'wakes' up and is ready for force tests. If the fly is given a small piece of paper then it 'lands' on the paper and when pried away from the flying insect, it starts to fly again. (Fig. 8)

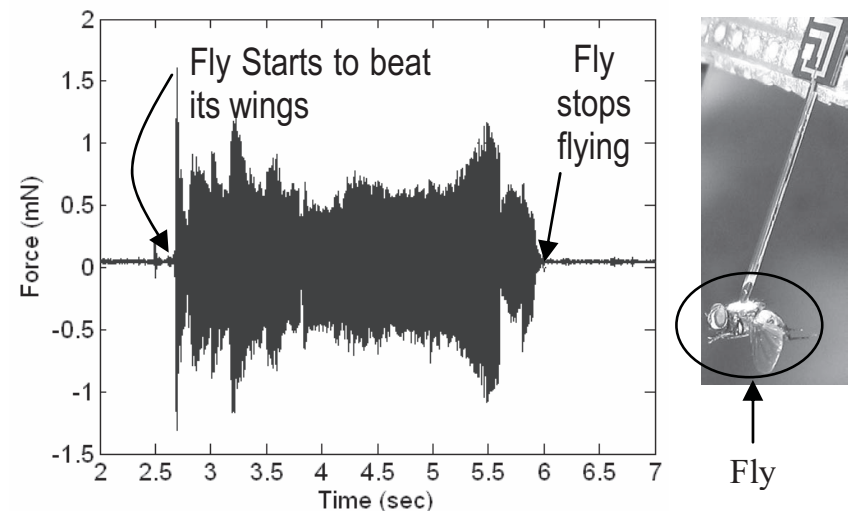

FIGURE 8: As the house fly beats its wings, the oscillating voltage across the strain gauge can be translated into the instantaneous forces using the calibration curves.

By taking the FFT of the signal we can look at the frequency content. The fundamental frequency for house flies is around $170 \mathrm{~Hz}$ in tethered flight. The reported wingbeat frequency of freeflying house flies is around $190 \mathrm{~Hz}$ (Fig. 9). The discrepancy between the two was also noted for free-flying and tethered locust and is probably a consequence of tethering [10]. Wingbeat frequency of many flying insects tends to deviate only slightly because beating wings at resonance minimizes the expended energy [8].

Using a stiffer force sensor (i.e. higher spring constant), insects with higher resonant frequencies and harmonics can also be detected. The force sensor can therefore be a very useful tool for measurement of flight forces in insects which beat their wings at a 
high enough frequency that it is very difficult to use high speed videography as there aren't enough frames per wingbeat.

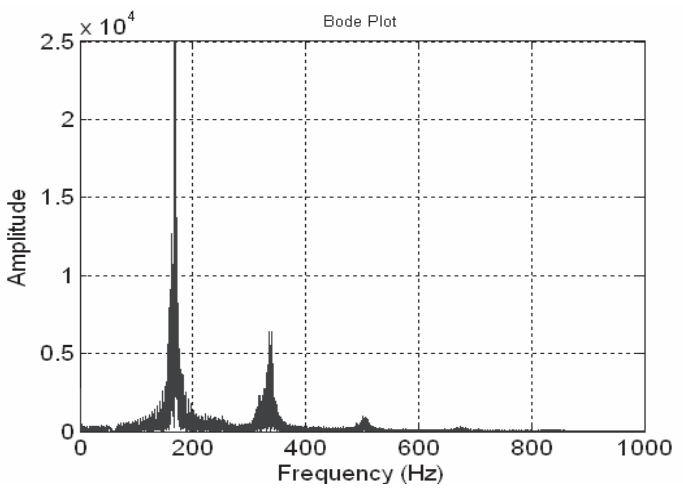

FIGURE 9: The frequency content of the time series flight force data reveals that for a house fly the fundamental mode is around $170 \mathrm{~Hz}$ and the first harmonic at roughly twice the fundamental mode.

The magnitude of forces applied by the fly change significantly over time and this shows that the fly is constantly modulating flight forces to make different maneuvers. The advance ratio per wingbeat is going to be smaller then estimated solely on the basis of instantaneous forces being measured since the fly experiences drag forces during upstroke.

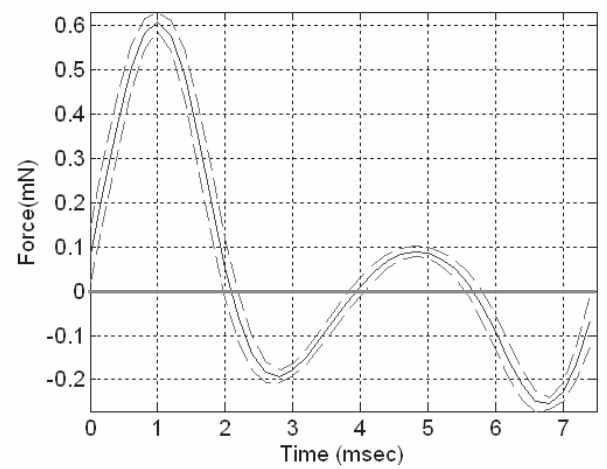

FIGURE 10: High resolution force response over one cycle shows the forces during down and up strokes. Data from 10 cycles has been averaged. The first force peak is due to the downstroke motion of the wings.

More importantly, tests with tethered flies have also been conducted to find forces generated during one upstrokedownstroke cycle (Fig. 10). Since the tethered insect produces flight forces in both horizontal and vertical directions, it is not possible to find out the relative contribution of lift, thrust and sideslip components with only one strain gauge. However, preliminary tests done in our lab using high speed videography of house flies tethered to the force sensor indicate that sideslip is not being actively controlled and therefore does not contribute significantly to instantaneous force measurement.

The force pattern like the one shown in fig. 10 varies widely depending on the kind of maneuver being performed by the fly. However, it can still be inferred that measured force is increasing during the downstroke which is responsible for most of the generated lift, while upstroke when the voltage is decreasing is associated with most of the forward thrust. The fact that force variation is not just a simple sinusoid clearly indicates the presence of complex mechanisms associated with unsteady aerodynamics.

\section{CONCLUSION}

A force sensor has been developed. The design of the sensor allows for measurement of instantaneous forces in tethered simulation of flight. The microfabricated sensor was calibrated for static and dynamic loads. Initial tests with tethered house flies have allowed measurement of instantaneous flight forces. Concurrent research on a multidirectional version of this force sensor will allow measurement of flight forces with more than one DOF. These quantitative force measurements when used in conjunction with high speed videography, will allow a better understanding of complex mechanisms underlining unsteady aerodynamics. In addition this tool will also allow for comparative biomechanical analysis of flight mechanism in different flying insects.

\section{REFERENCES}

[1] Vogel, S. "Flight in Drosophila II. Variations in stroke parameters and wing contour" J. exp. Biol. 46: pp:383-392 (1967)

[2] Wagner H. "Flight performance and visual control of flight of the freeflying housefly (Musca domestica)" Phil. Trans. R. Soc. Lond. B, 312: 581-595 (1983)

[3] D'Abrera, B. "Sphingidae mundi. Hawk moths of the world.” Faringdon, U.K.: E.W. Classey Ltd. (1986)

[4] Dickinson, M. H. and Lighton, J. R. B. "Muscle Efficiency and Elastic Storage in the Flight Motor of Drosophila" Science, Vol. 268, p. 87-90 (1995)

[5] Potassek DP, Sun Y, Fry SN and Nelson BJ. "Characterizing fruit fly flight behavior using a micro force sensor with a new comb drive configuration." J. of MEMS, Vol. 14, p 4-11. (2005)

[6] Nasir, M., Dickinson, M.,Bartsch and Liepmann, D., "Multidirectional Force and Torque Sensor for Insect Flight Research" Proc. of Transducers, p. 555-558 (2005)

[7] Bartsch, M. S., Partridge, A., Pruitt, B. L., Full. R. J. and Kenny, T. W. "A Three-Axis Piezoresistive Micromachined Force Sensor for Studying Cockroach Biomechanics." MEMS, ASME, Vol. 2, p. 443-448 (2000)

[8] Dudley, R "Biomechanics of Insect Flight" Princeton University Press, Princeton NJ, pp. 89-90, (2000).

[9] M Rockstein and PL Bhatnagar, "Duration and frequency of wing beat in the aging house fly, Musca domestica L", Biol Bull, Vol. 131: 479-486 (1966)

[10] Baker P.S., Gewecke M. and Cooter R.J., "The natural flight of the migratory locust, Locusta migratoria L. III. Wing-beat frequency, flight speed and attidude." J. comp Physiol A, Vol. 141, pp:233-237 (1981)

\section{ACKNOWLEDGEMENT}

The researchers would like to thank Packard Foundation for the funding of this project and Jae-Hyung Lim for help with data acquisition software. 